\title{
Actions et politiques publiques dans l'agriculture : libéralisation de l'économie, diversification des approches
}

\author{
Philippe Lacombe ${ }^{1}$ et Claude Napoléone ${ }^{2}$ \\ 1 Économiste, INRA, 75007 Paris, France \\ 2 Économiste, INRA, unité Écodéveloppement, 84914 Avignon, France
}

\begin{abstract}
Mots-clés :
développement agricole ; globalisation ; résistances endogènes innovations ; politiques agricoles ; coordination économique

Résumé - Le monde agricole, en raison de l'effet de ses pratiques sur l'environnement ou la santé humaine, comme de la relation de la société à l'usage du territoire qui l'environne, va être confronté à des choix publics contraignants sur les formes d'agriculture ou la nature de l'usage du sol. L'ignorer permet de repousser l'échéance mais accroîtra l'impact de la décision lorsqu'elle sera prise. Il nous paraît donc opportun d'élargir notre champ de vision. L'interprétation de l'économie agricole ne procède plus des seules considérations sectorielles mais doit s'appuyer sur d'autres éléments qui structurent les sociétés : l'alimentation, la santé, l'usage des territoires, la finance, le marché du travail, la conservation de l'environnement... Dans cette perspective, trois axes de recherche sont proposés : l'appréhension des rôles de l'agriculture coordonnée à d'autres secteurs d'activité ; l'analyse des conditions sociales de formation des attentes vis-à-vis de l'agriculture et les critères d'élaboration d'un choix public; la proposition d'instruments d'interventions. Il s'agit de développer des lectures du monde centrées sur la diversité (des acteurs, des structures sociales et de leurs relations aux institutions publiques). Une option serait de s'intéresser au fonctionnement des arènes politiques ou des marchés en tant que faits sociaux et d'étoffer une analyse critique des propositions ou dispositifs institutionnels nationaux et supranationaux.
\end{abstract}

\section{Keywords:}

agricultural development; globalization; endogenous resistance; innovation; agricultural policy; economic coordinating framework

\begin{abstract}
Actions and public policies in agriculture: economic liberalization requires diversified approaches. Because of its impact on the environment and on human health agriculture will be faced with constraining public trade-offs in its practices and land-use forms. Ignoring this may stretch the deadline but will increase the cost of necessary choices when these must be implemented. Therefore we need to widen our approach. Analyses of agricultural economy can no longer be tackled on a sectoral basis but must take in other social elements such as foods, health, land-use change, international finance, labor markets, environmental conservation, etc. In this context, three kinds of research avenues are proposed: investigation of the roles of agriculture as coordinated with other sectors; analysis of the social background which shapes expectations regarding agriculture and the criteria leading to the elaboration of public choice; proposals for policy tools. This approach is based on diversity (of actors, of social structures and their relations with public institutions). One option would be to focus on the functioning of markets and political arenas considered as social facts and to broaden a critical analysis of national and supranational institutional proposals and structures.
\end{abstract}

La libéralisation économique du secteur agricole amorcée à la fin du $X X^{\mathrm{e}}$ siècle entraîne de profonds changements dans l'organisation et le fonctionnement des agricultures du monde. Ces changements sont soutenus par des politiques publiques fondées sur une confiance optimiste dans le fonctionnement efficace et vertueux des marchés. Or, les krachs alimentaires récurrents amènent à tempérer cet optimisme sans, pour autant, l'éliminer. Dans ce cadre, le colloque de Cerisy ${ }^{1}$ nous a amenés à discuter de l'agriculture et de l'alimentation dans un

\footnotetext{
Auteur correspondant : philber.lacombe@aliceadsl.fr

1 Colloque «Agricultures et alimentations dans un monde globalisé », organisé à Cerisy-la-Salle en septembre 2011. Vous trouverez dans ce texte entre parenthèses des références à des interventions qui ont eu lieu pendant le colloque. Les enregistrements audio et/ou les diaporamas de ces interventions sont consultables sur les sites internet du Cirad (cerisy2011.cirad.fr) et d'Agropolis International (cerisy2011.agropolis.fr). Voir aussi, à propos de ce colloque, dans ce même numéro, l'introduction (Caron et al.) et les trois autres textes de synthèse (Dorin et al., Bricas et al., Hubert et al.).
} 
monde globalisé et, plus particulièrement à nous interroger sur les approches à promouvoir pour « quantifier, analyser et interpréter " les transformations contemporaines.

\section{Une situation nouvelle et « inattendue »}

La libéralisation économique en cours tend à conformer le secteur agricole aux règles contemporaines du commerce international : élimination des dispositifs protectionnistes, des soutiens à la production ou aux prix, des barrières à l'entrée de nouveaux acteurs économiques... Il s'ensuit des recompositions importantes dans la nature et la localisation des productions ou des marchés : les agricultures du monde doivent fonctionner dans un espace économique élargi du fait de l'extension des échanges marchands de denrées, mais également de techniques, d'intrants (avec l'action des firmes d'amont et d'aval), de travail (sous l'effet des migrations) et plus récemment de la terre (Jean Paul Charvet, Université Paris Ouest Nanterre la Défense ; Michel Foucher, Institut des hautes études de défense nationale). À côté d'une agriculture de survie peu insérée dans le commerce international, est visée la disparition progressive des exploitations familiales plus ou moins modernisées, au profit d'une industrialisation de l'agriculture non socialement régulée. Cela concourt à une banalisation de systèmes et pratiques agricoles, inhérente à une confiance dans les mécanismes les plus standards du marché concurrentiel : la libéralisation économique doit générer une croissance des productions et des échanges marchands, supposés améliorer l'approvisionnement alimentaire. La libéralisation génère conjointement l'entrée en scène de nouveaux acteurs souvent éloignés du secteur agricole ${ }^{2}$, plus puissants que lui et capables de prescrire des pratiques ou des dispositifs. Les acteurs anciens et nouveaux doivent donc se coordonner entre partenaires inégaux. Ils le font par la concurrence, en bannissant toute intervention de l'État jugée distorsive, c'est-à-dire en ne traitant pas, par l'action publique, les fonctions sociales attachées à l'agriculture : la dimension stratégique de l'approvisionnement alimentaire qui a pourtant motivé des politiques agricoles nationales, le choix d'un modèle de production (l'exploitation familiale) ou de commercialisation (la coopération) qui a orienté la forme des soutiens publics à l'agriculture ${ }^{3} .$.

\footnotetext{
2 Que ce soient des institutions financières sur les marchés des denrées, des États à travers des acquisitions foncières stratégiques ou encore la société dans son ensemble à travers de nouvelles normes encadrant l'agriculture.

3 Le refus de l'intervention publique concerne le champ du fonctionnement des marchés et non la création de règles (politique de concurrence ou sanitaire, normes de qualité, normalisations...).
}

Toutefois, les conjonctures erratiques des échanges marchands, en dépit des performances agricoles ou des quantités produites, et les manifestations violentes qui ont quelquefois suivi (les émeutes de la faim en 2008, par exemple) rappellent que le recours au marché n'évite pas les hausses de prix, les pénuries, les obstacles aux échanges, les paniques... Elles ont pour le moins donné une acuité nouvelle à la question de l'accès à l'alimentation et les problématiques agraires réinvestissent le débat social au point de devoir être traitées par les instances nationales ou internationales ${ }^{4}$. Elles encouragent également des mouvements d'opposition ou des propositions alternatives concernant tantôt les systèmes de production $^{5}$ et/ou de consommation ${ }^{6}$, tantôt les modes $\mathrm{d}^{\text {'échange }}{ }^{7}$. L'insertion des agricultures dans cet espace économique élargi fait donc naître un enjeu public ${ }^{8}$ tenant à la stabilité sociale et génère des débats traversant la société ou la recherche académique (Hervieu, 2002 ; Griffon, 2006 ; Pisani, 2007 ; Guillou et Matheron, 2011 ; Muller, 2011 ; Williamson, 2000...). Or, l'interprétation de l'économie agricole doit dépasser les seules considérations sectorielles, tant l'évolution de l'agriculture est commandée par des relations qui transcendent les notions d'aval ou d'amont, d'accès au crédit ou de diversification des activités, pour s'appuyer sur d'autres éléments qui structurent les sociétés : l'alimentation, la santé, l'usage des territoires, la finance, le marché du travail, la conservation de l'environnement... Dans cette perspective, les communications du colloque ont mis en lumière la diversité des formes sociales de production et de distribution, des modes de régulation, des acteurs ou des technologies de par le monde. Longtemps considérée comme archaïque ou transitoire, la diversité des systèmes est aujourd'hui traitée de manière plus positive (Ward Anseeuw, Cirad ; Estelle Bienabe, Cirad ; Perrine Burnod, Cirad ; Pierre Gasselin, Inra; Renato Maluf, Universidade Federal Rural do Rio de Janeiro ; François Traoré, Union nationale des producteurs de coton du Burkina Faso ; Geneviève Nguyen-Thole, École nationale supérieure agronomique de Toulouse [ENSAT] ; François Purseigle, ENSAT ; Marie-Claude Maurel, EHESS... entre autres), comme une richesse susceptible, sur le plan opérationnel, de participer à l'aménagement du territoire et, sur le plan analytique, d'induire un renouvellement de nos représentations et catégorisations (Bertrand Hervieu,

\footnotetext{
4 Ministère de l'Agriculture et de la Pêche, 2007 ; FAO, 2009 ; OECD-FAO, 2010...

5 L'agriculture biologique, par exemple.

6 Le mouvement slow food.

7 Les AMAP (Associations pour le maintien d'une agriculture paysanne) relèvent de ces propositions alternatives.

8 En entendant par là, la transformation d'une question locale, marginale, en une question pour laquelle une solution n'existe qu'à une échelle supérieure de type intervention publique ou organisation collective.
} 
ministère de l'Agriculture, Bernard Hubert, Inra, et Patrick Caron, Cirad).

Toutefois, nous n'assistons pas pour autant à l'émergence de nouvelles références intellectuelles qui auraient permis de proposer et de justifier des solutions plus complètes. Les actions publiques ayant fait suite aux conjonctures erratiques des marchés des denrées ou aux émeutes de la faim ont confirmé les orientations en cours en recherchant leur amélioration (diffusion de l'information, gestion des crises, mécanismes d'assurance, incorporation accrue de progrès techniques...). Comment expliquer une telle prégnance des positions libérales? Faut-il y voir un aveuglement idéologique (Stiglitz, 2010) ? Faut-il y voir un intérêt stratégique des acteurs les plus influents dans l'arène des négociations, générant des positions contraires aux déclarations fondatrices des grandes organisations internationales d'après-guerre (Supiot, 2010) ? Il s'agit, en tout cas, de nous interroger sur la place et le fonctionnement des agricultures dans le système économique global en ce début du $\mathrm{XXI}^{\mathrm{e}}$ siècle (Benoît Daviron, Cirad ; Éric Sabourin, Cirad ; Philippe Lacombe, Inra ; Henri Hocdé, Cirad).

\section{Les agricultures et le système économique mondial}

\section{Agricultures et politiques libérales}

Les politiques agricoles contemporaines font référence au marché dans l'optique des bienfaits attendus des théorèmes de l'économie du bien-être ${ }^{9}$. Or, la référence au marché s'est réalisée dans son acception la plus restrictive, c'est-à-dire indépendamment du contexte social. L'État ne disparaît pas, son rôle se modifie: il abandonne les références organisationnelles et sociales, d'équité ou de justice par exemple, pour un rôle de régulateur en dernière instance de la concurrence dont l'initiative est laissée aux entrepreneurs. D'où un paradoxe apparent: en doctrine, il est souhaité des agricultures market oriented alors qu'en pratique, le système ne fonctionne qu'avec une forte intervention de l'État pour institutionnaliser la concurrence. Différent du «laisser faire » du libéralisme standard, le néolibéralisme

\footnotetext{
9 1/ Pour une dotation en ressources donnée (i.e. une répartition des richesses donnée), une situation de concurrence pure et parfaite conduit à un optimum de Pareto (il n'existe pas de meilleure solution mutuellement plus avantageuse pour les individus y participant).

2/ Il existe plusieurs optimums puisqu'il existe plusieurs répartitions possibles des ressources : l'action publique doit choisir l'optimum préférable et donc agir sur les allocations initiales des agents pour l'atteindre.
}

actuel $^{10}$ associe un État fort à une économie libre (Gamble, 1988). De plus, en intervenant dans la fixation des conditions de la production, il «conduit les conduites » (en reprenant Foucault) et généralise un paradigme idéologique dont les défenseurs se trouvent être les acteurs insérés dans le système, y compris les moins privilégiés. L'extension de la rationalité marchande génère donc une "universalité » de la concurrence en faisant oublier que le marché comme les règles d'échanges ne sont pas «naturels"; ce sont des construits sociaux émanant d'une vision du monde particulière (Jacques Rémy, Inra). Pour les producteurs, l'alternative revient à être performants à l'aune des critères de concurrence ou être exclus du secteur... Alternative éprouvante lorsque l'acte de production n'a pas pour objet principal l'amélioration de la position concurrentielle ou la croissance des taux marginaux de profit ${ }^{11}$ ! Dès lors, des mouvements sociaux sont prévisibles ainsi que des formes de résistance et des innovations, potentiellement accessibles pour des entreprises individuelles ou des consommateurs non totalement contraints par les dispositifs publics d'accompagnement de la production.

\section{Résistances, innovations et renouvellements}

L'agriculture conserve des marges d'autonomie du fait d'une structure sectorielle encore dispersée qui lui permet de rechercher, en son sein, des formes de résistance ou d'adaptation. À titre d'exemple, l'agriculture biologique s'est construite depuis le début du $X X^{e}$ siècle à partir d'innovations développées en dehors de la recherche et des accompagnements publics. Ses propositions techniques et son accueil par la demande concourent maintenant à l'évolution de normes de production qui s'imposent au secteur dans son ensemble. Formellement, les formes de résistance ou d'innovations sont variées et peuvent correspondre à un strict encadrement public des échanges ou des prix de produits socialement stratégiques (denrées alimentaires de base), au soutien des formes d'organisation non pleinement concurrentielles (à titre de redistribution, de réciprocité, d'équité

\footnotetext{
${ }^{10}$ La notion de système néolibéral est ambiguë. Elle a des sens différents, parfois même contradictoires. Elle est parfois utilisée (notamment dans les discours partisans), comme l'apogée du « laisser faire ", de la défense inconditionnelle du marché et du jeu d'intérêts individuels. En ce qui nous concerne, nous utilisons ce terme pour caractériser une organisation fondée sur le marché et la concurrence à partir desquels sont gérées les relations au sein de la société, qu'elles soient individuelles ou collectives (comme les interventions de l'État) (Dardot et Laval, 2009 ; Audier, 2012 ; Dostaler, 2009 ; Audard, 2009).

11 C'est le cas des exploitations familiales vendant leurs surplus. La concurrence des agricultures industrialisées insérées dans les échanges mondiaux leur a fermé l'accès au marché domestique par ailleurs nécessaire à l'équilibre budgétaire de la famille.
} 
entre producteurs, de bénéfices non marchands, d'autoconsommation...) ou encore à l'instauration de normes sociales et environnementales incorporant les effets des solutions technologiques industrielles à l'évaluation de la qualité des produits (Yuna Chiffoleau, Inra ; Claire Lamine, Inra). Longtemps ignorées, méprisées ou refusées, ces formes déviantes par rapport au modèle standard de modernisation ont acquis une légitimité technique, économique et sociale. Elles trouvent aujourd'hui une place à côté de l'agriculture " conventionnelle » (parfois, elles la remplacent) et contribuent à la diversité des systèmes productifs ( $c f$. le texte de Dorin et al. dans ce numéro).

Toutefois, il y a encore un certain niveau de contradiction à penser le secteur agricole marchand comme inefficient au regard de critères sociaux ou environnementaux et à proposer des évolutions reposant sur ses résistances endogènes. La responsabilité des agriculteurs dans leurs choix de techniques ou de commercialisation n'est pas pleine et entière. Il existe un chemin de dépendance de la production agricole borné par les industries amont et aval ainsi que par les dispositifs publics, qui limitent les choix d'évolution des producteurs. Il y a donc la nécessité d'un choix social et politique complémentaire (ou englobant), qui doit être traité en tant que tel. À ce titre, la question agricole dans l'arène politique déborde les frontières traditionnelles du secteur agricole. Elle met en lumière les liens entre les formes de production et d'échange, la santé, la sécurité alimentaire, le cadre de vie, le changement climatique, l'énergie... Si des expérimentations endogènes au secteur agricole peuvent exister et si des préférences sociales s'expriment sur les formes productives ou d'échange, la généralisation des innovations les plus souhaitables interpelle les pouvoirs publics et la recherche académique dans leur ensemble ${ }^{12}$ (Michel Griffon, ANR \& Cirad ; Hervé Guyomard, Inra ; JeanChristophe Debar, AgriUS; Philippe Boullet, CERFRANCE ; Frédéric Goulet, Cirad).

\section{Enjeux et nouvelles problématiques de recherche}

Les changements dans la genèse des politiques publiques et le contenu des actions afférentes ont des conséquences sur la conception des recherches en sciences sociales à leur sujet. D'une part, l'espace géographique et social concerné s'élargit et se complexifie. D'autre part, les formes actuelles de l'enchâssement de

12 L'agroécologie propose, à ce titre, un cadre d'analyse tenant à la désectorialisation de l'agriculture... tout du moins l'agroécologie qui prend en charge la question sociale en tant que telle (Gliessman, 2007 ; Altieri, 1989). l'agriculture dans la société et l'environnement incitent à reconsidérer l'efficience des approches sectorielles ou technicistes (Louis-Georges Soler, Inra). Dès lors, comment penser l'élaboration de choix publics dans de telles situations évolutives tendant à globaliser les dynamiques et les enjeux ? Il est, certes, possible de déterminer des solutions économiquement optimales en ignorant les structures sociales et les processus historiques. Toutefois, à côté de ces approches fragiles sur le long terme, il nous semble important de développer des lectures du monde plus centrées sur la diversité des acteurs, des structures sociales et de leurs relations aux institutions publiques. Une option serait, notamment, de caractériser les conditions d'émergence de décisions publiques socialement légitimes, au sein de relations entre partenaires différents et inégaux, de formes d'organisation multiples issues d'une histoire plurielle et détentrice de légitimités parfois contradictoires. À notre sens, le monde agricole, en raison de l'effet de ses pratiques sur l'environnement ou la santé humaine, comme de la relation de la société au territoire qui l'environne, va inéluctablement être confronté à des choix publics contraignants sur les formes d'agriculture ou la nature de l'usage du sol. L'ignorer permet de repousser l'échéance mais accroîtra l'impact de la décision lorsqu'elle sera prise. Il nous parait donc opportun d'élargir le champ de vision et de promouvoir un dialogue pluraliste, nous situant dans le champ des compromis. Cette diversification des problématiques appelle un enrichissement des thématiques de recherche, amplement justifié par la littérature scientifique et l'actualité économique ; trois axes peuvent être proposés :

- L'appréhension des rôles de l'agriculture coordonnée à d'autres secteurs d'activité. Il s'agit d'éclairer un débat social en précisant la nature des diverses visions de la re-conception de l'agriculture, ses conditions d'évolution, les conflits qui peuvent en émaner et les conséquences prévisibles de leur mise en œuvre (Claude Napoléone, Inra). Si l'agriculture se « désectorialise » en développant des liens avec d'autres domaines d'activités, le marché peut s'avérer défaillant pour traiter, seul, de ces fonctionnalités multiples ${ }^{13}$. En revanche, l'évaluation des services rendus par chacune de ces activités conjointes devient nécessaire pour une correcte coordination par l'action publique. L'univers des possibles est plus grand que celui qu'on envisage actuellement et mérite d'être exploré par la recherche publique.

- L'analyse des conditions sociales de formation des attentes vis-à-vis de l'agriculture et les critères d'élaboration d'un choix public (Nicolas Bricas, Cirad). Les coordinations entre l'agriculture et le reste de l'économie procèdent d'un marché, d'une organisation ou d'une

13 La quasi-absence d'internalisation des effets des pratiques productives (agricoles comme industrielles) sur l'environnement est le meilleur exemple. 
forme hybride. L'émergence d'une forme particulière de coordination résulte d'un rapport social en mesure d'imposer sa rationalité aux logiques concurrentes. Il faut donc considérer les critères de hiérarchisation des attentes sociales propres à chaque groupe concurrent ; ils ne sont pas neutres et ont des significations qui doivent être confrontées entre elles pour éclairer le choix public $^{14}$.

- La proposition d'instruments d'interventions. L'évolution des politiques agricoles induit une modification des instruments d'intervention utilisés. Certains deviennent obsolètes (dans un cadre de libéralisation, les instruments jugés distorsifs), d'autres sont actualisés pour devenir compatibles avec de nouveaux objectifs, d'autres encore doivent être créés (en matière d'environnement ou de sécurité sanitaire). Or, ces évolutions ne sont, également, pas neutres : le système d'incitations évoluant, les intérêts se déplacent et les bénéficiaires peuvent changer, rappelant ainsi la portée sociopolitique de tout instrument d'action publique. La proposition de nouveaux instruments ou d'évolutions possibles des instruments actuels est un champ de recherche important, dans l'optique d'un compromis pérenne sur l'agriculture.

\section{Conclusion}

L'espace de fonctionnement des agricultures du monde se transforme, d'un capitalisme aménagé dans le cadre national à un système globalisé dessinant un État fort pour une économie libre. Dans ce cadre, les politiques contemporaines régulant la production et les échanges agricoles génèrent des inégalités économiques, sociales et politiques dans la répartition des ressources, ainsi que des mouvements sociaux attachés à la pénurie. Dans un régime de concurrence généralisée entre agricultures hétérogènes, à côté des systèmes les plus rentables, certains types d'agriculture sont condamnés à disparaître, tandis que d'autres ne survivront que grâce aux subventions que la société acceptera de leur distribuer (lorsqu'elle le peut)... En outre, à l'instar d'un système bancaire "Too Fat To Fall», les impératifs de stabilité sociale, notamment au titre de la sécurité alimentaire, amèneront à tolérer ou même à soutenir les systèmes productifs ayant généré l'instabilité : dans le

\footnotetext{
14 À titre d'exemple, une politique retenant la notion d'irréversibilité des effets environnementaux des différents usages d'un sol (la stérilisation par des pratiques intensives répétées, la destruction d'un écosystème non reproductible, l'urbanisation d'une terre productive...) ordonnerait les critères d'efficience des usages d'une manière fort différente que ne le font, d'un côté, les modèles agronomiques intensifs et, de l'autre, les modèles de maximisation de la rente urbaine ou de l'offre de logements.
}

cas des émeutes de la faim de 2008, les États n'ont pu retrouver le calme social qu'en subventionnant l'importation de denrées issues du marché mondial, c'est-à-dire issues du modèle industriel qui a déstabilisé le système agricole traditionnel en militant pour une suppression des subventions taxées alors de distorsion de concurrence... En corollaire, les expériences localisées tendant à proposer des voies de transition vers des systèmes sociotechniques respectueux de préférences sociales ou écologisés, mais moins performants économiquement, sont bridées par les règles du marché « libre ». La libéralisation en cours des échanges ne sature toutefois pas l'espace social : il y a des alternatives qui, bien que dispersées, peuvent ouvrir un pluralisme d'analyses. Il est toujours possible de s'intéresser au fonctionnement des arènes politiques ou des marchés en tant que faits sociaux et, dans cette perspective, il est souhaitable d'étoffer une analyse critique des propositions ou dispositifs institutionnels nationaux et supranationaux.

Si l'on veut nourrir neuf milliards de personnes en 2050, des progrès techniques dans l'agriculture seront nécessaires mais ils n'auront pas d'effets sans changer de paradigme : la production agricole actuelle suffirait à nourrir la planète alors que des gens meurent de faim par défaut d'accès aux denrées (Paillard et al., 2010). Audelà de la performance du système $\mathrm{d}$ 'allocation de la ressource, les États sont en mesure (et même en charge) d'édicter des préférences sociales globales via les organisations internationales (la référence au « travail décent » proposée par le Bureau international du travail, l'enjeu alimentaire au sens de la $\mathrm{FAO}$, la biodiversité après Rio...). Or, les préoccupations d'organisation sociale largement associées dans le passé aux actions économiques sont absentes ou peu présentes dans les politiques contemporaines régulant la production et les échanges agricoles. Il est vrai que l'engagement dans une voie de coordination entre politiques et entre États éloignerait d'une gestion par le marché au profit d'une gestion par l'organisation, c'est-à-dire une coordination des politiques agricoles maximisant les complémentarités entre zones et minimisant les conséquences sociales et environnementales des systèmes productifs favorisés. Dans le domaine de la coordination ${ }^{15}$, l'actualité ne rend pas optimiste... Toutefois, en dehors de solutions extrêmes (collectivisation versus marché généralisé), nul doute que des accommodements peuvent être apportés aux dynamiques libérales en cours : produire autant que possible, échanger autant que nécessaire... N'est-ce pas le rôle des politiques publiques de concevoir et de gérer des compromis? N'est-ce pas le rôle de la recherche publique de fournir les connaissances éclairant les choix publics?

\footnotetext{
15 Échange d'information sur les productions, les stocks, les investissements, la gestion concertée de l'offre...
} 


\section{Références}

Altieri, M.A., 1989. Agroecology: A new research and development paradigm for world agriculture, Agric., Ecosyst. E Env, 27, 37-46.

Audard, C., 2009. Qu'est-ce que le libéralisme? Éthique, politique, société, Paris, Gallimard.

Audier, S., 2012. Néo-libéralisme(s), une archéologie intellectuelle, Paris, Grasset.

Dardot, P., Laval, C., 2009. La nouvelle raison du monde. Essai sur la société néolibérale, Paris, La Découverte.

Dostaler, G., 2009. Les chemins sinueux de la pensée libérale, L'Économie politique, 44.

FAO, 2009. Comment nourrir le monde en 2050. Rapport.

Gamble, A., 1988. The free economy and the strong state: the politics of Tatcherism, Macmillan.

Gliessman, S.R., 2007. Agroecology: the ecology of sustainable food systems, New York, CRC Press, Taylor \& Francis.

Griffon, M., 2006. Nourrir le monde, Paris, Odile Jacob.

Guillou, M., Matheron, G., 2011. 9 milliards d'hommes à nourrir, Paris, François Bourin.
Hervieu, B., 2002. Le développement durable : une nécessité pour nourrir le monde, Dossier de l'environnement de l'INRA, 27, 7-22.

Ministère de l'Agriculture et de la Pêche, 2007. Objectif terres 2020 : pour un nouveau modèle agricole français. Rapport, http://terres2020.agriculture.gouv.fr/.

Muller, P., 2011. Les politiques publiques, Paris, PUF.

OECD-FAO, 2010. Agricultural outlook 2010-2019. Rapport.

Paillard, S., Treyer, S., Dorin, B. (Eds), 2010. Agrimonde. Scénarios et défis pour nourrir le monde en 2050, Versailles, Quæ.

Pisani, E., 2007. Une politique mondiale pour nourrir le monde, Springer.

Stiglitz, J., 2010. Le triomphe de la cupidité, Paris, Les liens qui libèrent.

Supiot, A., 2010. L'esprit de Philadelphie : la justice sociale face au marché total, Paris, Éditions du Seuil.

Williamson, J., 2000. What should the World Bank think about the Washington Consensus? The World Bank Research Observer, 15, 4. 